\title{
Binomial Identities and Moments of Random Variables
}

\section{José A. Adell and Alberto Lekuona}

Abstract. We give unified simple proofs of some binomial identities, by using an elementary identity on moments of random variables.

1. INTRODUCTION. The starting point of this note is the following binomial identity

$$
\sum_{k=0}^{n}\left(\begin{array}{l}
n \\
k
\end{array}\right) \frac{(-1)^{k}}{r+k}=\frac{n !}{r(r+1) \cdots(r+n)},
$$

valid for any $r>0$. Peterson [7] gave a proof of (1) and a generalization of it (Identity 2 below) using conditional expectations involving the exponential distribution. Nakata [6] provided a short proof of (1) using the minimum of a finite sequence of independent and uniformly distributed random variables. Recently, Spivey [8] has developed a more classic probabilistic approach to prove (1) and its generalization based on a standard balls-and-jars interpretation.

The aim of this note is to show that identity (1), and many other binomial identities of a similar nature, can be derived in a unified way from elementary identities between moments of suitable random variables stated in formula (2) below.

The basic concepts of probability theory used here are continuous random variables and their moments. The probability density $\rho(x)$ of a continuous random variable $T$ is a nonnegative function whose integral from $-\infty$ to $\infty$ is 1 . Given a nonnegative integer $n$, the moment of order $n$ of $T$ is the mathematical expectation of $T^{n}$, i.e.,

$$
E T^{n}=\int_{-\infty}^{\infty} x^{n} \rho(x) d x
$$

whenever this integral exists. For general moments of arbitrary random variables, we refer the reader to the classical book by Billingsley [2, pp. 273-280].

Suppose now that $T$ is a continuous random variable whose moments of order $s$, $E T^{s}, r-1 \leq s \leq r+n-1$, are finite. By the binomial formula, we obviously have the following identity between the moments of $T$

$$
\sum_{k=0}^{n}\left(\begin{array}{l}
n \\
k
\end{array}\right)(-1)^{k} E T^{r+k-1}=E T^{r-1}(1-T)^{n} .
$$

It turns out that every choice of the random variable $T$ in (2) gives us a different binomial identity. Of course, the most interesting cases are those in which we can compute in closed form the right-hand side in (2). In addition, formula (2) allows us to write in integral form the corresponding binomial identity, depending on the probability density of the random variable $T$ (in this respect, see Identity 2 below). We finally mention that formula (2) is also true for arbitrary random variables $T$. 
Many authors have used moments of random variables as an efficient tool to deal with various different problems coming from combinatorics and classical analysis. For instance, Chang and $\mathrm{Xu}$ [3] have obtained a Chu-Vandermonde multivariate formula by computing the moment of order $n$ of the chi-square random variable with $m$ degrees of freedom. Vignat and Moll [13] have shown that a variety of classical binomial identities, including some generalizations of the Chu-Vandermonde formula, can be obtained by using moments of finite sums of independent random variables. Representations of the Bernoulli, Euler and Gegenbauer polynomials in terms of moments of appropriate random variables can be found in Sun [11] and Srivastava and Vignat [9]. Finally, Ta [12] has recently introduced a nice probabilistic approach to Appell polynomials by connecting them to moments of random variables.

We will use the following notations. For any real $\alpha$, it is understood that

$$
\left(\begin{array}{l}
\alpha \\
n
\end{array}\right)=\frac{\alpha(\alpha-1) \cdots(\alpha-n+1)}{n !} .
$$

For any $p>0$, Euler's gamma function is defined as

$$
\Gamma(p)=\int_{0}^{\infty} x^{p-1} e^{-x} d x .
$$

When $n$ is a nonnegative integer, $\Gamma(n+1)=n$ ! and $0 !=1$. For any $p>0$ and $q>0$, we recall that

$$
\int_{0}^{1} x^{p-1}(1-x)^{q-1} d x=\beta(p, q)=\frac{\Gamma(p) \Gamma(q)}{\Gamma(p+q)}
$$

where $\beta(\cdot, \cdot)$ is Euler's beta function, as well as

$$
\frac{\Gamma(p+n)}{n ! \Gamma(p)}=\left(\begin{array}{c}
p-1+n \\
n
\end{array}\right)
$$

2. BINOMIAL IDENTITIES. To illustrate how formula (2) works, we will make three choices of the random variable $T$ in it, thus obtaining three binomial identities. The first two were considered by Peterson [7] and Spivey [8] and involve uniformly distributed random variables, whereas the third one involves a random variable having the beta density.

Identity 1. For any $r>0$, we have

$$
\sum_{k=0}^{n}\left(\begin{array}{l}
n \\
k
\end{array}\right) \frac{(-1)^{k}}{r+k}=\frac{n !}{r(r+1) \cdots(r+n)} .
$$

Proof. Let $U$ be a random variable having the uniforms distribution on $(0,1)$. It readily follows from (3) that

$$
E U^{r+k-1}=\frac{1}{r+k}, \quad E U^{r-1}(1-U)^{n}=\frac{\Gamma(r) \Gamma(n+1)}{\Gamma(r+n+1)} .
$$

Therefore, the conclusion follows by setting $T=U$ in formula (2). 
Identity 2. For any $r>0$ and $j=1,2, \ldots$, we have

$$
\begin{aligned}
\sum_{k=0}^{n}\left(\begin{array}{l}
n \\
k
\end{array}\right) \frac{(-1)^{k}}{(r+k)^{j}} & =\frac{1}{r\left(\begin{array}{c}
r+n \\
n
\end{array}\right)} \sum_{0 \leq k_{1} \leq \ldots \leq k_{j-1} \leq n} \frac{1}{\left(r+k_{1}\right)\left(r+k_{2}\right) \cdots\left(r+k_{j-1}\right)} \\
& =\int_{0}^{1} x^{r-1}(1-x)^{n} \frac{(-\log x)^{j-1}}{(j-1) !} d x .
\end{aligned}
$$

Proof. Let $\left(U_{i}, 1 \leq i \leq j\right)$ be a finite sequence of independent and identically distributed random variables having the uniform distribution on $(0,1)$, and denote by $T_{i}=U_{1} \cdots U_{i}, 1 \leq i \leq j$. We will show Identity 2 by choosing $T=T_{j}$ in formula (2). In fact, since the random variables under consideration are independent and identically distributed, we see that

$$
E T_{j}^{r+k-1}=E U_{1}^{r+k-1} \cdots E U_{j}^{r+k-1}=\frac{1}{(r+k)^{j}} .
$$

For $i=1, \ldots, j$, we consider the function

$$
f_{i}(n)=\left(\begin{array}{c}
r-1+n \\
n
\end{array}\right) E T_{i}^{r-1}\left(1-T_{i}\right)^{n}, \quad n=0,1,2, \ldots,
$$

and claim that

$$
f_{i}(n)=\frac{1}{r+n} \sum_{k=0}^{n} f_{i-1}(k), \quad n=0,1,2, \ldots, i=2, \ldots, j .
$$

Actually, since we can write $T_{i}=T_{i-1} U_{i}$ as the product of two independent random variables, we have from (3)

$$
\begin{aligned}
E T_{i}^{r-1}\left(1-T_{i}\right)^{n} & =E\left(U_{i} T_{i-1}\right)^{r-1}\left(1-U_{i}+U_{i}\left(1-T_{i-1}\right)\right)^{n} \\
& =\sum_{k=0}^{n}\left(\begin{array}{l}
n \\
k
\end{array}\right) E U_{i}^{r+k-1}\left(1-U_{i}\right)^{n-k} E T_{i-1}^{r-1}\left(1-T_{i-1}\right)^{k} \\
& =\sum_{k=0}^{n}\left(\begin{array}{l}
n \\
k
\end{array}\right) \frac{\Gamma(r+k) \Gamma(n-k+1)}{\Gamma(r+n+1)} E T_{i-1}^{r-1}\left(1-T_{i-1}\right)^{k} \\
& =\frac{n ! \Gamma(r)}{\Gamma(r+n+1)} \sum_{k=0}^{n} f_{i-1}(k) .
\end{aligned}
$$

This, together with (4) and definition (6), shows claim (7). On the other hand, it is easily checked from (3) and (4) that

$$
f_{1}(m)=\left(\begin{array}{c}
r-1+m \\
m
\end{array}\right) E U_{1}^{r-1}\left(1-U_{1}\right)^{m}=\frac{1}{r+m}, \quad m=0,1,2, \ldots
$$

Thus, claim (7) allows us to use induction in order to obtain

$$
f_{j}(n)=\frac{1}{r+n} \sum_{0 \leq k_{1} \leq \cdots \leq k_{j-1} \leq n} \frac{1}{\left(r+k_{1}\right)\left(r+k_{2}\right) \cdots\left(r+k_{j-1}\right)} .
$$


The first equality in Identity 2 follows by choosing $T=T_{j}$ in formula (2) and taking into account (5), (6), and (8). Finally, the probability density of the random variable $T_{j}$ is given by (see, for instance, Feller $[4$, p. 26])

$$
\rho_{j}(x)=\frac{(-\log x)^{j-1}}{(j-1) !}, \quad 0<x<1 .
$$

As a consequence, the second equality in Identity 2 follows by writing in integral form the right-hand side in formula (2) with $T=T_{j}$.

It is worth noting that products of independent and uniformly distributed random variables are interesting probabilistic tools in certain areas of number theory and mathematical analysis. Among other instances, we mention the application of such products to obtain integral representations of the Riemann zeta function and identities of series involving the Stirling numbers of the first kind (see [10]), as well as applications to establish rates of convergence for the iterates of Cesàro operators (see [1]).

Identity 3. For any real numbers $p$ and $q$, we have

$$
\sum_{k=0}^{n}(-1)^{k}\left(\begin{array}{c}
p-1+k \\
k
\end{array}\right)\left(\begin{array}{c}
p+q-1+n \\
n-k
\end{array}\right)=\left(\begin{array}{c}
q-1+n \\
n
\end{array}\right) .
$$

Proof. Since the identity is a polynomial equation in $p$ and $q$, it suffices to show the result for $p>0$ and $q>0$. Let $T$ be a random variable having the beta density (see $[4$, p. 50])

$$
\rho(x)=\frac{x^{p-1}(1-x)^{q-1}}{\beta(p, q)}, \quad x \in(0,1), \quad p, q>0 .
$$

For $k, n=0,1, \ldots$, we see from (3) that

$$
E T^{k}=\frac{\beta(p+k, q)}{\beta(p, q)}, \quad E(1-T)^{n}=\frac{\beta(p, q+n)}{\beta(p, q)} .
$$

Hence, applying formula (2) to the case at hand with $r=1$, we get

$$
\sum_{k=0}^{n}\left(\begin{array}{l}
n \\
k
\end{array}\right)(-1)^{k} \beta(p+k, q)=\beta(p, q+n) .
$$

This is nothing else but Identity 3 , as follows by using (3), (4), and a bit of algebra.

ACKNOWLEDGMENT. The authors would like to thank the referees and the Editors for their careful reading of the manuscript and for their suggestions, which greatly improved the final outcome. This work was supported by research grants MTM2015-67006-P, DGA (E-64), and by FEDER funds.

\section{REFERENCES}

1. J. A. Adell, A. Lekuona, Rates of convergence for the iterates of Cesàro operators, Proc. Amer. Math. Soc. 138 (2010) 1011-1021.

2. P. Billingsley, Probability and Measure, Wiley Series in Probability and Mathematical Statistics, John Wiley \& Sons, New York, 1995 
3. G. Chang, C. Xu, Generalization and probabilistic proof of a combinatorial identity, Amer. Math. Monthly 118 (2011) 175-177.

4. W. Feller, An Introduction to Probability Theory and Its Applications. Vol. II, John Wiley \& Sons, Inc., New York, 1971.

5. N. L. Johnson, S. Kotz, N. Balakrishnan, Continuous Univariate Distributions. Vol. 2. Wiley Series in Probability and Mathematical Statistics: Applied Probability and Statistics, John Wiley \& Sons, New York, 1995.

6. T. Nakata, Another probabilistic proof of a binomial identity, Fibonacci Quart. 52 (2014) 139-140.

7. J. Peterson, A probabilistic proof of a binomial identity, Amer. Math. Monthly 120 (2013) 558-562.

8. M. Z. Spivey, Probabilistic proofs of a binomial identity, its inverse, and generalizations, Amer. Math. Monthly 123 (2016) 175-180.

9. H. M. Srivastava, C. Vignat, Probabilistic proofs of some relationships between the Bernoulli and Euler polynomials, Eur. J. Pure Appl. Math. 5 (2012) 97-107.

10. P. Sun, Product of uniform distribution and Stirling numbers of the first kind, Acta Math. Sin. (Engl. Ser.) 21 (2005) 1435-1442.

11. P. Sun, Moment representation of Bernoulli polynomial, Euler polynomial and Gegenbauer polynomials, Statist. Probab. Lett. 77 (2007), 748-751.

12. B. Q. Ta, Probabilistic approach to Appell polynomial, Expo. Math. 33 (2015) 269-294.

13. C. Vignat, V. H. Moll, A probabilistic approach to some binomial identities, Elem. Math. 70 (2015) $55-66$.

JOSÉ A. ADELL Departamento de Métodos Estadísticos. Universidad de Zaragoza. 50009 Zaragoza (Spain).

adell@unizar.es

ALBERTO LEKUONA Departamento de Métodos Estadísticos. Universidad de Zaragoza. 50009 Zaragoza (Spain).

lekuona@unizar.es 